\title{
Circulating SFRP1 promoter methylation status in gastric adenocarcinoma and esophageal squamous cell carcinoma
}

\author{
CHANG LIU* ${ }^{*}$ NAN LI* ${ }^{*}$ HENG LU, ZHENGKAI WANG, CHUNYAN CHEN, \\ LIN WU, JIONG LIU, YOUKE LU and FANGYU WANG
}

Department of Gastroenterology and Hepatology, Jinling Hospital, School of Medicine, Southern Medical University, Nanjing, Jiangsu 210002, P.R. China

Received October 25, 2014; Accepted November 11, 2014

DOI: $10.3892 /$ br.2014.388

\begin{abstract}
The secreted frizzled-related protein 1 (SFRP1) gene plays an important role in carcinogenesis of digestive system cancer. Previous studies proved that circulating DNA promoter methylation may be a suitable biomarker for cancer patients. The aim of the present study was to investigate whether the promoter methylation status of serum SFRPl is a potential biomarker for gastric adenocarcinoma (GAC) and esophageal squamous cell carcinoma (ESCC). The blood samples obtained from $42 \mathrm{GAC}$ and 36 ESCC patients were detected for the promoter methylation status of SFRP1 by methylation-specific polymerase chain reaction. The control group included 42 benign gastrointestinal disease volunteers ( 24 benign gastric disease and 18 benign esophageal disease) and 20 healthy volunteers. Serum SFRP1 methylation was evident in $30.95 \%$ (13/42) GAC patients and $38.89 \%(14 / 36)$ ESCC patients, which is clearly higher compared to $8.33 \%$ $(2 / 24)$ in benign gastric disease, $11.11 \%(2 / 18)$ in benign esophageal disease and $5 \%(1 / 20)$ in healthy volunteers $(\mathrm{P}<0.05)$. The association between the serum SFRPI promoter methylation status and the clinical pathological features were further analyzed and methylation of the SFRPI gene was significantly associated with age $>60$ years in GAC patients $(\mathrm{P}=0.027)$. However, no correlations between the SFRPI methylation status and other clinicopathological parameters were found. In conclusion, the $S F R P 1$ promoter was detected frequently in the serum of GAC and ESCC patients. The detection of circulating methylated SFRPI in the serum may be a useful biomarker for upper gastrointestinal cancer patients.
\end{abstract}

Correspondence to: Professor Fangyu Wang, Department of Gastroenterology and Hepatology, Jinling Hospital, School of Medicine, Southern Medical University, 305 East Zhongshan Road, Nanjing, Jiangsu 210002, P.R. China

E-mail: wangfangyu1965@163.com

${ }^{*}$ Contributed equally

Key words: methylation, SFRP1 gene, gastric adenocarcinoma, methylation-specific polymerase chain reaction, esophageal squamous cell carcinoma

\section{Introduction}

Gastric adenocarcinoma (GAC) and esophageal squamous cell carcinoma (ESCC) are two major causes of cancer-related fatalities in China. Although gastroscopy is considered the most sensitive screening tool for upper gastrointestinal cancers, it remains limited in attributing to its potential risk, high cost and intolerance of the invasive procedure in patients (1). Therefore, reliable noninvasive tests for screening and diagnostic purposes are required.

Accumulating evidence indicates that carcinogenesis is regulated and controlled not only by genetic but also by epigenetic changes $(2,3)$. There is a marked acceleration observed in the past decade validating the concept that cancer is a disease of epigenetic alterations that are leading candidates for the development of specific markers for cancer detection, diagnosis and prognosis (4). As the most common molecular epigenetic change in human cancer, DNA methylation was proved to silence the tumor suppressor genes in $\operatorname{GAC}(5,6)$ and ESCC (7). Due to the relatively significant circulating DNA quantity in peripheral blood, analyses of methylation in serum DNA can potentially serve as an excellent tumor marker for diagnosis and prognosis of cancer (8). The secreted frizzled-related protein 1 ( $S F R P 1)$ gene belongs to the family of five secreted glycoproteins that have been identified as modulators of the Wnt signaling pathway involved in the development of cancer (9). Previous studies have shown that SFRPI is downregulated by promoter methylation in numerous types of digestive system cancer, which makes it a candidate for tumor gene suppression (10-12).

The present study attempted to identify the SFRPI promoter methylation status in serum from patients with GAC and ESCC by methylation-specific polymerase chain reaction (MSPCR), compared to benign gastrointestinal disease and healthy volunteers. The correlation between serum SFRP1 gene promoter methylation and patient clinical pathological parameters were also analyzed to further evaluate the clinical significance of this epigenetic molecular change.

\section{Materials and methods}

Study population. All the samples from human subjects were collected following the approval of the study by the Ethical 
Table I. SFRP1 sequences of the primers used in MSPCR.

\begin{tabular}{llcr}
\hline Gene & \multicolumn{1}{c}{ Primer sequence (5'-3') } & Product size, bp & Annealing temperature, ${ }^{\circ} \mathrm{C}$ \\
\hline $\mathrm{U}$ & F: 5'-GTAGTTTTTGGAGTTAGTGTTGTGT-3' $^{\prime}$ & 126 & 56 \\
& R: 5'-ACCTACAATCAAAAACAACACAAACA-3' & 119 \\
M & F: 5'-GTTTTCGGAGTTAGTGTCGCGC-3' & & 58 \\
& R: 5'-ACGATCGAAAACGACGCGAACG-3'
\end{tabular}

SFRP1, secreted frizzled-related protein 1; MSPCR, methylation-specific polymerase chain reaction; bp, basepair; U, unmethylated; M, methylated; F, forward; R, reverse.

Committee of Jinling Hospital (Nanjing, Jiangsu, China) and informed consent was obtained from all the patients. The 140 blood samples were obtained from 42 GAC patients, 36 ESCC patients, 42 benign upper gastrointestinal disease patients ( 24 benign gastric disease and 18 benign esophageal disease, such as chronic gastritis, gastric ulcer, benign polyp, nonmalignant adenoma and gastroesophageal reflux disease; data not shown) and 20 healthy volunteers. Based on pathologic evidence, which were from gastroscopy, all the patients were diagnosed at the Department of Gastroenterology and Hepatology of Jinling Hospital between August 1, 2011 and November 30, 2012. All the patients and healthy volunteers were genetically unrelated and were Han Chinese.

Sample collection. Each patient donated $5 \mathrm{ml}$ of peripheral venous blood collected from 1 day after the patients were admitted to the hospital. At this time point, the patients did not start their treatment (surgery and/or chemo-radiation therapy). All the blood samples were kept in tubes containing clot activator at $4^{\circ} \mathrm{C}$ for $2 \mathrm{~h}$ and samples were centrifuged at $\sim 2,000 \mathrm{x} \mathrm{g}$ for $10 \mathrm{~min}$ to isolate sera. Twenty serum samples from healthy volunteers were obtained from the Blood Center of Jinling Hospital and were used as normal controls. All the serum samples were stored at $-80^{\circ} \mathrm{C}$ until use.

DNA extraction and MSPCR. Serum genomic DNA, extracted by the Axygen blood mini kit (Axygen, Union City, CA, USA) according to the manufacturer's instructions, was stored at $-80^{\circ} \mathrm{C}$ until use. The serum DNA was modified with sodium bisulfite using the DNA methylation kit (Sigma, St. Louis, MO, USA), also according to the manufacturer's instructions. The methylation status of the $\mathrm{CpG}$ islands in the promoter region of SFRPI was determined by MSPCR in GAC, ESCC, benign gastrointestinal disease and healthy control samples. Two sets of SFRPI primers, described elsewhere (13), were used to discriminate between the methylated and unmethylated alleles. MSPCR was determined with two-step amplification/detection MSP instructions, as in previous studies $(8,10,13,14)$. Briefly, the PCR mixture contained 6.25 $\mu \mathrm{l}$ AmpliTaq Gold 360 Master mix (Applied Biosystems, Foster City, CA, USA), $0.5 \mu \mathrm{mol}$ of each primer (Invitrogen, Shanghai, China), $3.25 \mu \mathrm{l}$ modified DNA and was adjusted by double-distilled $\mathrm{H}_{2} \mathrm{O}$ to a final volume of $25 \mu \mathrm{l}$. The cycling conditions consisted of one incubation for 2 min at $94^{\circ} \mathrm{C}$, followed by 38 cycles of a 30 -sec denaturation at $94^{\circ} \mathrm{C}$, a $30-\mathrm{sec}$ anneal (unmethylation at $56^{\circ} \mathrm{C}$, methylation at $58^{\circ} \mathrm{C}$ ), a $45-\mathrm{sec}$ extension at $72^{\circ} \mathrm{C}$ and a final extension at $72^{\circ} \mathrm{C}$ for $7 \mathrm{~min}$. The PCR products were electrophoresed through $2 \%$ agarose gels, stained with ethidium bromide and visualized with ultraviolet illumination. Table I lists the sequences of the PCR primers, products size and annealing temperature. All the experiments were performed in duplicate.

Statistical analysis. All the data were analyzed by the SPSS 17 software (SPSS, Inc., Chicago, IL, USA). Statistical analysis was performed using the $\chi^{2}$ and Fisher's exact tests. Two-sided tests were used to determine significance and $\mathrm{P}<0.05$ was considered to indicate a statistically significant difference.

\section{Results}

SFRP1 promoter methylation status in all the patients and controls. The methylation status of the SFRPI promoter in serum DNA from 42 GAC, 36 ESCC, 24 benign gastric disease, 18 benign esophageal disease patients and 20 healthy volunteers was successfully performed. The representative agarose gel electrophoresis results are shown in Fig. 1. As shown, there were three statuses of the methylation analysis result: Complete methylation (only the methylated SFRPI promoter was amplified), incomplete methylation (methylated and unmethylated $S F R P 1$ promoter were amplified) and unmethylation (only the unmethylated $S F R P 1$ promoter was amplified). Complete and incomplete methylation were defined as the methylation status. As a result, serum SFRPI promoter methylation was detected in 13 GAC, 14 ESCC, two benign gastric disease, two benign esophageal disease patients and one healthy volunteer. The detection frequencies of serum SFRPI promoter methylation was $30.95 \%$ in GAC and $38.89 \%$ in ESCC, which were significantly higher than those in the benign gastric disease $(8.33 \%, \mathrm{P}<0.05)$, benign esophageal disease $(11.11 \%, \mathrm{P}<0.05)$ and healthy volunteers $(5.00 \%, \mathrm{P}<0.05)$, respectively.

Correlation between the serum RASSF1A promoter methylation and clinicopathological parameters in patients with GAC and ESCC. The association between the serum SFRP1 promoter methylation status and clinicopathological parameters were analyzed in the GAC and ESCC patients. The results are listed in Table II. As indicated, methylation of the SFRPl gene was significantly associated with age $>60$ years in the GAC patients $(\mathrm{P}=0.027)$. However, in the 
Table II. Correlation between the serum SFRP1 methylation gene promoter methylation status and clinicopathological parameters in gastric adenocarcinoma and esophageal squamous cell carcinoma patients.

\begin{tabular}{|c|c|c|c|c|c|c|c|c|c|c|}
\hline \multirow[b]{2}{*}{ Characters } & \multicolumn{5}{|c|}{ Gastric adenocarcinoma } & \multicolumn{5}{|c|}{ Esophageal squamous cell carcinoma } \\
\hline & No. & M & $\mathrm{U}$ & $\chi^{2}$ & P-value & No. & M & $\mathrm{U}$ & $\chi^{2}$ & P-value \\
\hline Total no. & 42 & 13 & 29 & & & 36 & 14 & 22 & & \\
\hline \multicolumn{11}{|l|}{ Age, years } \\
\hline$<60$ & 17 & 2 & 15 & $4.92^{\mathrm{a}}$ & $0.027^{\mathrm{a}, \mathrm{b}}$ & 23 & 8 & 18 & $7.882^{\mathrm{a}}$ & $0.140^{c}$ \\
\hline$>60$ & 25 & 11 & 14 & & & 13 & 6 & 4 & & \\
\hline \multicolumn{11}{|l|}{ Gender } \\
\hline Male & 30 & 8 & 22 & $0.902^{\mathrm{a}}$ & $0.463^{\mathrm{c}}$ & 25 & 8 & 17 & $1.634^{\mathrm{a}}$ & $0.273^{\mathrm{c}}$ \\
\hline Female & 12 & 5 & 7 & & & 11 & 6 & 5 & & \\
\hline \multicolumn{11}{|l|}{ Differentiation } \\
\hline Well/moderate & 16 & 4 & 12 & $0.428^{\mathrm{c}}$ & $0.733^{\mathrm{a}}$ & 21 & 9 & 12 & $0.334^{\mathrm{a}}$ & $0.563^{\mathrm{a}}$ \\
\hline Poor/UN & 26 & 9 & 17 & & & 15 & 5 & 10 & & \\
\hline \multicolumn{11}{|l|}{ Stage } \\
\hline $\mathrm{I} / \mathrm{II}$ & 9 & 2 & 7 & $0.408^{\mathrm{c}}$ & $0.695^{\mathrm{a}}$ & 24 & 6 & 15 & $2.258^{\mathrm{a}}$ & $0.133^{\mathrm{a}}$ \\
\hline III/IV & 33 & 11 & 22 & & & 12 & 8 & 7 & & \\
\hline \multicolumn{11}{|l|}{ Metastasis } \\
\hline Yes & 15 & 4 & 11 & $0.278^{\mathrm{a}}$ & $0.734^{\mathrm{c}}$ & 13 & 3 & 10 & $2.141^{\mathrm{a}}$ & $0.143^{\mathrm{a}}$ \\
\hline No & 27 & 9 & 17 & & & 23 & 11 & 12 & & \\
\hline \multicolumn{11}{|l|}{ CEA (ng/ml) } \\
\hline$>9.7$ & 18 & 7 & 11 & $0.928^{\mathrm{a}}$ & $0.335^{\mathrm{a}}$ & 6 & 4 & 2 & $2.338^{\mathrm{a}}$ & $0.181^{\mathrm{c}}$ \\
\hline$<9.7$ & 24 & 6 & 18 & & & 30 & 10 & 20 & & \\
\hline
\end{tabular}

${ }^{\mathrm{a}} \chi^{2}$ test; ${ }^{\mathrm{b}}$ statistically significant $(\mathrm{P}<0.05)$; ${ }^{\mathrm{F}}$ Fisher's exact test. SFRP1, secreted frizzled-related protein $1 ; \mathrm{M}$, methylated; U, unmethylated; UN, undifferentiated; CEA, carcinoembryonic antigen.

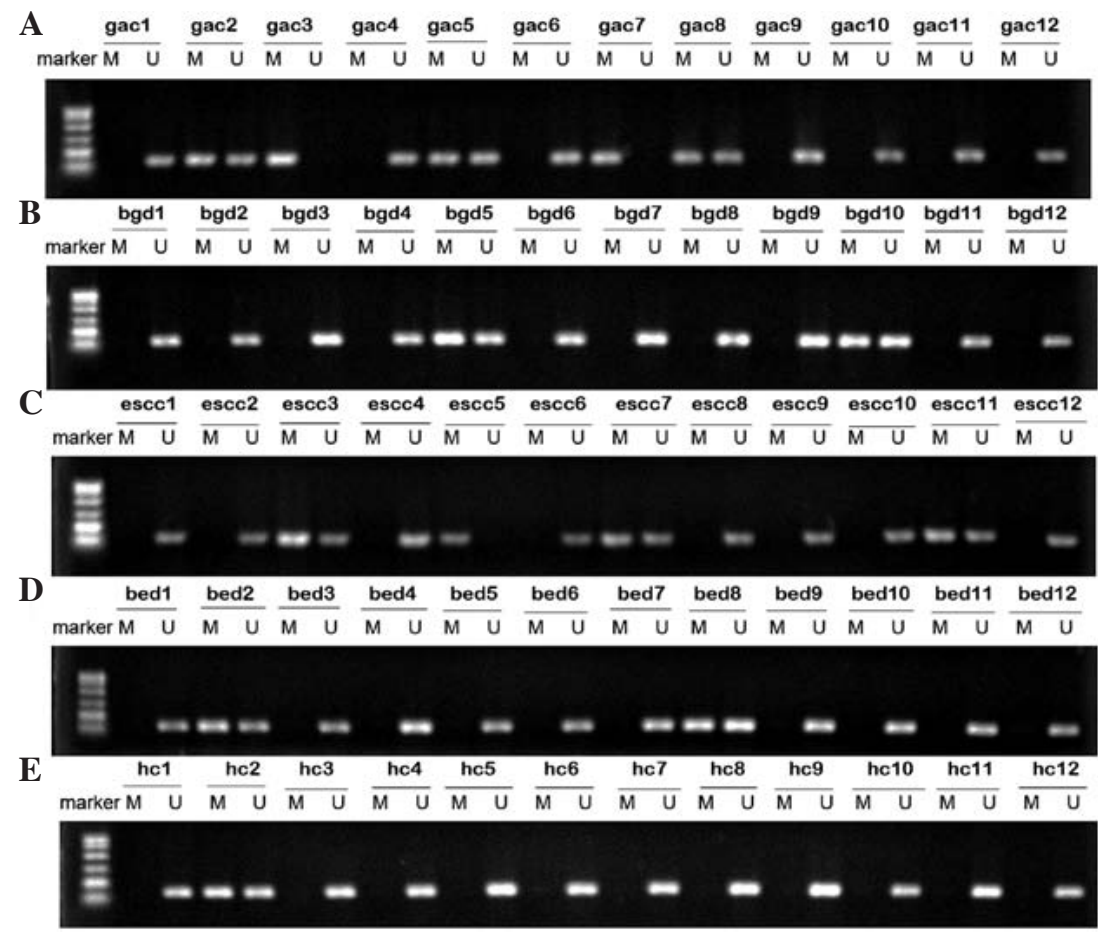

Figure 1. Representative results showing the SFRP1 promoter methylation status identified by MSPCR. Identification of the SFRP1 promoter methylation status in serum samples from (A) gastric adenocarcinoma patients, (B) benign gastric disease patients, (C) esophageal squamous cell carcinoma patients, (D) benign esophageal disease patients and (E) healthy control. Lanes $\mathrm{M}$ and $\mathrm{U}$ indicate the amplified products with primers recognizing specific methylated and unmethylated sequences, respectively. SFRP1, secreted frizzled-related protein 1; MSPCR, methylation-specific polymerase chain reaction; M, methylated; U, unmethylated; GAC, gastric adenocarcinoma; BGD, benign gastric disease; ESCC, esophageal squamous cell carcinoma; BED, benign esophageal disease; HC, healthy control. 
ESCC patients the difference was not significant $(\mathrm{P}=0.145)$. There was no other correlation between the SFPRI promoter methylation status and patient gender, clinical stage, tumor differentiation grade, metastasis or serum carcinoembryonic antigen levels.

\section{Discussion}

Patients with early stage upper gastrointestinal cancer have no typical disease-related symptoms, which is why numerous gastric and esophageal cancers are detected at an advanced stage, even with incurable distant metastases (15). Thus, there is an urgent requirement to identify the valuable markers for early diagnosis evaluation of these types of cancer.

Aberrant methylation of promoter DNA regions that are rich in $\mathrm{CpG}$ islands is the key step in epigenetic gene silencing (16). Molecular alterations of the epigenome, particularly DNA methylation, have emerged as alternative targets of biomarker research and exhibit potentially great clinical significance (3). In numerous types of cancers, SFRPI defects at the genetic (mutations) and epigenetic (transcriptional inactivation) levels have been reported (17). Epigenetically, SFRPI is inactivated via promoter methylation, and tumor suppressor genes are silenced in cancers (18).

In the present study, the arbitrary promoter region methylation in $S F R P 1$ was frequently observed in the serum of GAC and ESCC patients. The frequency of SFRP1 methylation was $30.92 \%$ in the serum of GAC patients, clearly higher compared to $8.33 \%$ in benign gastric disease $(\mathrm{P}=0.035)$ and $5 \%$ in healthy volunteers $(\mathrm{P}=0.018)$. The frequency is higher when compared to $11.3 \%$, reported in the study by Tahara et al (5) that used quantitative MSPCR. However, Guo et al (19) detected the methylation of $S F R P 1$ in gastric cancer tissue and obtained the frequency of $78.7 \%$ (74/94). The difference may result from the different methods and sample sizes. Another possibility is that in certain clinical cases, the methylation of tumor suppressor genes may be exhibited in tumor tissue, but have not been detected in serum (14). In 2005, the study by Zou et al (11) detected the mRNA expression of the SFRP genes and quantified by quantitative reverse transcription PCR in esophageal adenocarcinoma cell lines, and found that $S F R P I$ was detected in $93 \%$ of 40 cancers tissue and $81 \%$ of 37 Barrett's epithelia. This indicates that aberrant promoter methylation results in downregulation of the SFRP gene expression and occurs commonly in Barrett's esophagus and the early stage of esophageal cancer. Subsequently, Liu et al (20) detected hypermethylation of the promoter of SFRP1 using plasma DNA from 81 ESCC patients, with a percentage of $29.6 \%$, but the study did not compare the result with benign esophageal disease. The present study had a similar, but higher, result of $38.89 \%$ in 36 ESCC patients. SFRP1 methylation was also detected in the benign esophageal disease with a frequency of $11.11 \%$, which is markedly lower compared to in ESCC patients $(\mathrm{P}=0.035)$. These above findings confirmed that aberrant promoter methylation of SFRPI may be an early event in the development of gastric and esophageal cancer and it can be a candidate biomarker for population screening. When it is detected in benign upper gastrointestinal disease patients and the healthy population, this may be a warning to perform gastroscopy and provide attention to this.
In the present study, the serum SFRPI promoter methylation status was further compared to the clinicopathological parameters. As with a number of previous studies, no association was found between serum SFRPI methylation and patient gender, tumor differentiation grade and distal metastasis in these two types of cancers. However, in GAC, methylation of the SFRPI gene was significant associated with age $>60$ years in GAC patients $(\mathrm{P}=0.027)$. The similar result was reported by Wang et al (21) in which the age was observed to be significantly associated with the methylation status of the $S F R P 1$ gene in breast cancer tissue. Age is an important risk factor and the association between DNA methylation and aging has been reported constantly $(2,5,22,23)$. As early as 1987, Wilson et al (22) found that a significant loss of DNA 5-methyldeoxycytidine residues in old age disrupt the cellular gene expression and contribute to the physiological decline of the animal. Teschendorff et al (23) also found that the age-associated methylation signature is present in preneoplastic conditions and may drive gene expression changes associated with carcinogenesis. To verify the association of the SFRP1 gene methylation and age, this requires a large group of clinic observation and follow-up. The research regarding the biological mechanism of SFRPI promoter methylation and age is also noteworthy to GAC.

The present study had limitations, such as the small sample size and lack of follow-up due to the limited time constraint. Thus, whether SFRPI is a diagnostic, progressive, predictive or prognostic biomarker remains unknown. However, the sensitive and specific detection of the SFRPI methylation patterns makes it a choice of biomarker for the clinical management of cancer patients. These investigations will continue in our future study.

In conclusion, the serum promoter methylation of the SFRPI gene can be frequently detected in GAC and esophageal carcinoma patients and it may be a promising biomarker for such cancers. In general, more prospective studies are required to prove the use as a biomarker and to use it to aid in the clinical decision-making. Therefore, investigating a larger study population may reveal more significant results.

\section{Acknowledgements}

The present study was supported by the National Natural Science Foundation of China (grant no. 81270453) to Fangyu Wang, the National Natural Science Foundation of China (grant no. 81302162) to Nan Li and Jinling Hospital Foundation (grant no. 2011052) to Chang Liu.

\section{References}

1. Tanzer M, Liebl M and Quante M: Molecular biomarkers in esophageal, gastric, and colorectal adenocarcinoma. Pharmacol Ther 140: 133-147, 2013.

2. Qu Y, Dang S and Hou P: Gene methylation in gastric cancer. Clin Chim Acta 424: 53-65, 2013.

3. How Kit A, Nielsen HM and Tost J: DNA methylation based biomarkers: practical considerations and applications. Biochimie 94: 2314-2337, 2012.

4. Baylin SB and Jones PA: A decade of exploring the cancer epigenome - biological and translational implications. Nat Rev Cancer 11: 726-734, 2011.

5. Tahara T, Maegawa S, Chung W, et al: Examination of whole blood DNA methylation as a potential risk marker for gastric cancer. Cancer Prev Res (Phila) 6: 1093-1100, 2013. 
6. Yao D, Shi J, Shi B, et al: Quantitative assessment of gene methylation and their impact on clinical outcome in gastric cancer. Clin Chim Acta 413: 787-794, 2012.

7. Ishii T, Murakami J, Notohara K, et al: Oesophageal squamous cell carcinoma may develop within a background of accumulating DNA methylation in normal and dysplastic mucosa. Gut 56: 13-19, 2007.

8. Wang YC, Yu ZH, Liu C, Xu LZ, Yu W, Lu J, Zhu RM, Li GL, Xia XY, Wei XW, et al: Detection of serum RASSF1A promoter hypermethylation in serum from gastric and colorectal adenocarcinoma patients. World J Gastroenterol 14: 3074-3080, 2008.

9. Jones SE and Jomary C: Secreted Frizzled-related proteins: searching for relationships and patterns. Bioessays 24: 811-820, 2002.

10. Nojima M, Suzuki H, Toyota M, et al: Frequent epigenetic inactivation of SFRP genes and constitutive activation of Wnt signaling in gastric cancer. Oncogene 26: 4699-4713, 2007.

11. Zou H, Molina JR, Harrington JJ, et al: Aberrant methylation of secreted frizzled-related protein genes in esophageal adenocarcinoma and Barrett's esophagus. Int J Cancer 116: 584-591, 2005

12. Bu XM, Zhao $\mathrm{CH}$ and Dai XW: Aberrant expression of Wnt antagonist SFRP1 in pancreatic cancer. Chin Med J (Engl) 121: 952-955, 2008

13. Urakami S, Shiina H, Enokida H, et al: Combination analysis of hypermethylated Wnt-antagonist family genes as a nove epigenetic biomarker panel for bladder cancer detection. Clin Cancer Res 12: 2109-2116, 2006.

14. Wang Y, Yu Z, Wang T, Zhang J, Hong L and Chen L: Identification of epigenetic aberrant promoter methylation of RASSF1A in serum DNA and its clinicopathological significance in lung cancer. Lung Cancer 56: 289-294, 2007.
15. Tani N, Ichikawa D, Ikoma D, et al: Circulating cell-free mRNA in plasma as a tumor marker for patients with primary and recurrent gastric cancer. Anticancer Res 27: 1207-1212, 2007.

16. Liu JB, Wu XM, Cai J, et al: $\mathrm{CpG}$ island methylator phenotype and Helicobacter pylori infection associated with gastric cancer. World J Gastroenterol 18: 5129-5134, 2012.

17. Urakami S, Shiina H, Enokida H, et al: Wnt antagonist family genes as biomarkers for diagnosis, staging, and prognosis of renal cell carcinoma using tumor and serum DNA. Clin Cancer Res 12: 6989-6997, 2006.

18. Mao W, Wordinger RJ and Clark AF: Focus on molecules: SFRP1. Exp Eye Res 91: 552-553, 2010.

19. Guo Y, Guo W, Chen Z, Kuang G, Yang Z and Dong Z: Hypermethylation and aberrant expression of Wnt-antagonist family genes in gastric cardia adenocarcinoma. Neoplasma 58: 110-117, 2011.

20. Liu JB, Qiang FL, Dong J, et al: Plasma DNA methylation of Wnt antagonists predicts recurrence of esophageal squamous cell carcinoma. World J Gastroenterol 17: 4917-4921, 2011.

21. Wang S, Dorsey TH, Terunuma A, et al: Relationship between tumor DNA methylation status and patient characteristics in African-American and European-American women with breast cancer. PLoS One 7: e37928, 2012.

22. Wilson VL, Smith RA, Ma S and Cutler RG: Genomic 5-methyldeoxycytidine decreases with age. J Biol Chem 262: 9948-9951, 1987.

23. Teschendorff AE, Menon U, Gentry-Maharaj A, et al: Age-dependent DNA methylation of genes that are suppressed in stem cells is a hallmark of cancer. Genome Res 20: 440-446, 2010 . 